Preprint typeset in JHEP style - PAPER VERSION

FNT-T/2004/03, SHEP-03-12, CAFPE-26/03

UG-FT-156/03, LAPTH-1028/04, BA-TH/495/04

\title{
Higgs boson self-couplings at the LHC as a probe of extended Higgs sectors
}

\author{
M. Moretti \\ Dipartimento di Fisica - Università di Ferrara and INFN - Sezione di Ferrara, Via \\ Paradiso 12, 44100 Ferrara, Italy
}

\section{S. Moretti \\ School of Physics 83 Astronomy, University of Southampton, Highfield, Southampton SO17 1BJ, UK. E-mail: stefano@hep.phys.soton.ac.uk \\ F. Piccinini \\ INFN - Sezione di Pavia and Dipartimento di Fisica Nucleare e Teorica, via Bassi 6, 27100 Pavia, Italy}

\section{*R. Pittau}

Departamento de Física Teórica y del Cosmos and Centro Andaluz de Física de Partículas Elementares (CAFPE), Universidad de Granada, E-18071 Granada, Spain

\section{${ }^{\dagger}$ A.D. Polosa \\ LAPTH, BP 110, Chemin de Bellevue, 74941 Annecy-le-Vieux Cedex, France}

ABSTRACT: A study of two-Higgs-doublet models (2HDM) in the decoupling limit reveals the existence of parameter configurations with a large triple-Higgs self-coupling as the only low-energy trace of the departure from a Standard Model (SM) Higgs sector. This observation encourages attempts to search for double Higgs production at the Large Hadron Collider (LHC) even in mass regions which have been shown to be very hard to probe in the context of SM-like Higgs self-couplings. In this document we consider the case of an Intermediate Mass Higgs (IMH) boson, with $120 \mathrm{GeV} \lesssim M_{H} \lesssim 140 \mathrm{GeV}$, produced in pairs via vector-vector fusion, Higgs-strahlung and associate production with heavy-quarks and decaying into $b \bar{b}$ pairs. After a detailed signal-to-background analysis, we confirm that the observation of a Higgs-pair signal is very challenging in the framework of the SM, even at the LHC with upgraded luminosity. In contrast, we verify that the sensitivity is sufficient to detect departures from the SM which would escape detection in the measurements of the single-Higgs production channels.

Keywords: Electro-Weak Symmetry Breaking, Higgs potential, Hadron Colliders, Beyond the Standard Model.

\footnotetext{
* On leave of absence from: Dipartimento di Fisica Teorica, Torino and INFN Sezione di Torino, Italy

†Present address: Centro Studi e Ricerche "E. Fermi", via Panisperna 89/A, 00184 Roma, Italy
} 


\section{Contents}

1. Introduction 1

2. Higgs-pair production at the LHC 2

3. The decoupling limit of $2 \mathrm{HDMs} \quad 4$

4. Triple-Higgs self-couplings at the LHC 6

$\begin{array}{lll}4.1 & \text { Vector-vector fusion } & 7\end{array}$

4.2 Higgs-strahlung 8

4.3 Heavy-quark associate production 10

5. Discussion of the results 11

6. Summary and outlook $\quad 13$

\section{Introduction}

The ability of the ATLAS and CMS experiments at the LHC to detect a SM Higgs boson over the full range of allowed masses has now been established by several detailed studies [1]. According to the latter, a multitude of production and decay channels will be accessible and will enable the determination of several of the couplings of the Higgs boson with accuracies that, depending on the precise value of the Higgs mass, $M_{H}$, can be as good as $10 \%$ [1]. Possible significant departures from the SM expectations will allow to infer that the underlying Higgs sector is not as simple as postulated in the SM. However, there is no guarantee that non-SM Higgs sectors will become manifest via these measurements. Examples are given by 2HDMs, including Supersymmetric models, where the spectrum of Higgs bosons beyond the lightest one could be very heavy and the couplings of the latter to fermions and gauge bosons reduce to those of the SM. We explore in this paper the possibility that in this limit a large deviation from the SM value $\lambda_{H H H}^{(0)}$ of the triple-Higgs self-coupling involving the lightest Higgs state, hereafter $\lambda_{H H H}$, is allowed to survive. Such a circumstance would select the production of Higgs boson pairs as a possible channel for the identification of a non-SM Higgs structure.

In Section 2 we review known results about Higgs-pair production in the SM. In Section 3 we discuss the main features of the decoupling limit in 2HDMs and demonstrate the existence of parameter sets leading to strongly enhanced triple-Higgs self-couplings, while maintaining the deviations of the couplings to fermions and to gauge bosons below the sensitivity range of direct LHC measurements. We verified that for (some of) these models 
the analysis of Higgs-pair production can be performed rather reliably (for the channels we consider in the analysis) by simply rescaling in the Lagrangian the value of $\lambda_{H H H}^{(0)}$, keeping all other Higgs couplings fixed to their SM values ${ }^{1}$. Notice that this implies that our analysis is model independent ${ }^{2}$ : our conclusions apply to any extended Higgs sector in regions of parameter space where the only observable departure from the SM is a sizeable deviation of $\lambda_{H H H}$ from $\lambda_{H H H}^{(0)}$. In Section 4 we focus on the study of Higgs-pair production at the LHC for IMH bosons, namely with $120 \mathrm{GeV} \lesssim M_{H} \lesssim 140 \mathrm{GeV}$. In this region the detection of SM Higgs pairs is particularly challenging, due to the very large QCD backgrounds and the very small signal cross sections. We extend previous analyses by considering the production channels induced by vector-vector fusion, Higgs-strahlung and associated production with a top quark pair. For all these channels we assume the $H \rightarrow b \bar{b}$ decay mode and we perform a background study. In Section 5 we present our assessment of the prospects to constrain deviations from the SM expectations, both at the LHC and at the so-called SLHC [2], the tenfold luminosity increase option of the LHC. Section 6 contains our summary and conclusions.

\section{Higgs-pair production at the LHC}

The complete reconstruction of the Higgs potential necessarily requires the measurement of the Higgs self-couplings. These include a trilinear and a quartic interaction, parameterized by the coupling constants $\lambda_{H H H}$ and $\lambda_{H H H H}$, which in the SM take the following values:

$$
\lambda_{H H H}^{(0)}=-3 \frac{M_{H}^{2}}{v}, \quad \lambda_{H H H H}^{(0)}=-3 \frac{M_{H}^{2}}{v^{2}},
$$

where $v=246 \mathrm{GeV}$ is the Higgs doublet vacuum expectation value. A direct measurement of $\lambda_{\mathrm{HHH}}$ can be obtained via the detection of Higgs boson pairs, wherein a contribution is expected from the production of a single off-shell Higgs boson splitting into two. However, the corresponding graphs will always be accompanied by diagrams where the two Higgses are radiated independently, with strength proportional to the Yukawa or gauge couplings. As a result, different production mechanisms will lead to different sensitivities of the Higgspair production rates on the value of $\lambda_{H H H}$ (notice that $\lambda_{H H H}$ contributes, through loop radiative corrections, also to vector-boson propagators and vertices [3], giving however effects that are too tiny to be detected even in precision observables).

The leading production channels of Higgs boson pairs at hadron colliders [4]-[6] (see also [7]-[8]) are basically the same used for single-Higgs boson searches, namely:

$$
g g \rightarrow H H \text { ( } g g \text { fusion), } g g, q \bar{q} \rightarrow Q \bar{Q} H H \text { (heavy-quark associated production), }
$$

\footnotetext{
${ }^{1}$ For such channels this is due to the fact that, if we isolate the diagram which involves the triple-Higgs self-coupling, its contribution is a constant at high energies. Therefore, despite the fact that $\lambda_{H H H} \neq \lambda_{H H H}^{(0)}$ breaks gauge invariance, it is not crucial to enforce the unitarity of the cross section and it makes sense to assess the impact of changing the $\lambda_{H H H}$ value while keeping all other couplings fixed. We have explicitly verified, as discussed later, that this is indeed the case in the context of the 2HDM, namely, with a gauge invariant calculation.

${ }^{2}$ In this spirit, we will use the label $H$ to identify a light Higgs state in both the SM and a generic extension, while reserving the symbol $h$ specifically to the lightest Higgs boson of our example scenario, i.e., the $2 \mathrm{HDM}$ discussed in Section 3.
} 


$$
q q^{\left({ }^{\prime}\right)} \rightarrow q q^{\left({ }^{\prime}\right)} H H \text { (vector-vector fusion), } \quad q \bar{q}^{\left({ }^{\prime}\right)} \rightarrow V H H \text { (Higgs-strahlung), }
$$

wherein two Higgs bosons instead of one enter the final states and with $V=W^{ \pm}$or $Z$, $Q=b, t$ and $q^{\left({ }^{\prime}\right)}$ referring to any possible light (anti)quark flavor combinations ${ }^{3}$. The corresponding production rates are shown in Fig. 1 (borrowed from [7]), where we have superimposed those for heavy-quark associated production. The arrows indicate the variation in rate expected when changing the Higgs trilinear self-coupling in the range $\lambda_{H H H}^{(0)} / 2<$ $\lambda_{H H H}<3 / 2 \lambda_{H H H}^{(0)}$. Depending on the value of $M_{H}$, different decay channels dominate the final state [8]. For a so-called IMH boson, with $120 \mathrm{GeV} \lesssim M_{H} \lesssim 140 \mathrm{GeV}$, the decay phenomenology is dominated by the channel $H \rightarrow b \bar{b}$. For $M_{H} \gtrsim 140 \mathrm{GeV}$, the $H \rightarrow W^{ \pm(*)} W^{\mp}$ and $H \rightarrow Z^{(*)} Z$ modes share the largest fractions of the decay rate.

Given the rather low Higgs-pair production cross sections and the potentially large backgrounds associated with final states with the best decay rates (i.e., involving four $b$-quarks), naive arguments lead to the expectation that detection of IMH boson pairs via $g g \rightarrow H H \rightarrow b \bar{b} b \bar{b}$ within the SM is most probably not feasible at the LHC and very difficult at the SLHC [2]. This had been suggested already in Refs. [9, 10] (see also Ref. [11]) and later quantitatively confirmed in Ref. [12], where the production and decay channel $g g \rightarrow H H \rightarrow b \bar{b} \tau^{+} \tau^{-}$was shown to provide a better sensitivity to $\lambda_{H H H}$. The potential of the rarer signatures $g g \rightarrow H H \rightarrow b \bar{b} \gamma \gamma$ and $g g \rightarrow H H \rightarrow b \bar{b} \mu^{+} \mu^{-}$has been reviewed in Ref. [13], where the former was found to provide at the SLHC some limited scope in constraining $\lambda_{H H H}$ when $M_{H} \approx 120 \mathrm{GeV}$. For a heavier Higgs boson, $M_{H} \gtrsim 150 \mathrm{GeV}$, the situation is much brighter in comparison. In Ref. [14] it was found that it should be possible at the LHC with design luminosity to establish that the SM Higgs boson has a non-zero trilinear self-coupling and that the ratio $\lambda_{H H H} / \lambda_{H H H}^{(0)}$ can be restricted to the range $0-3.8$ at $95 \%$ confidence level (CL), by exploiting $g g \rightarrow H H$ production and decay via $H H \rightarrow W^{ \pm(*)} W^{\mp} W^{ \pm(*)} W^{\mp}$, in a variety of leptonic and/or hadronic final states. At the SLHC [2], such limits can be improved further and even $4-5 \sigma$ excesses can be established.

All such studies were based on the leading production channel of SM Higgs bosons, namely gluon-gluon fusion (see Fig. 1). We present here the first results of studies performed in the case of IMH boson pairs produced via the other three production modes in Eq. (2.2). The reason to exploit the latter is due to the additional triggers available in each case, with respect to the $g g \rightarrow H H$ mode: forward/backward jets in vector-vector fusion and high transverse-momentum leptons/light jets from gauge boson decays in both heavy-quark associated production and Higgs-strahlung. This could in principle enable one to perform additional independent measurements of $\lambda_{H H H}$ for an IMH boson, for which the current situation is extremely problematic. Although we will see that, as a result of our partonlevel study, the available statistics is in general too low for quantitative estimates of $\lambda_{H H H}^{(0)}$, we will show that there is room for a signal, or for non-trivial limits, in the context of the 2HDM discussed in the following Section.

\footnotetext{
${ }^{3}$ The case $Q=b$ in heavy-quark associated production is actually irrelevant in the SM. In a general 2HDM it may become significant. However, we will be interested only in the decoupling regime, which will be specified in more detail in the following Section. In this limit the departure of the cross section from the $\mathrm{SM}$ value is contrained to be at most within $70 \%$. Hence, we have ignored the case $Q=b$ altogether in our study.
} 


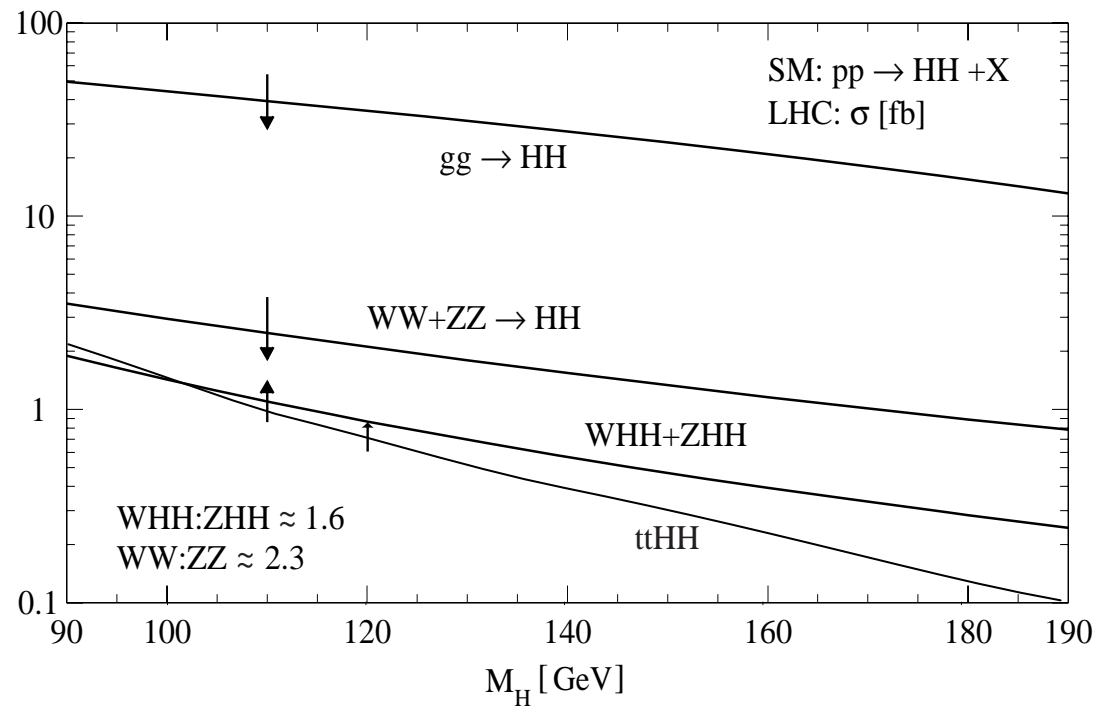

Figure 1: Cross sections for Higgs-pair production in the SM via gluon-gluon fusion, vector-vector fusion and Higgs-strahlung (from [7]), plus top-quark associated production. The vertical arrows correspond to a variation of $\lambda_{H H H}$ from $1 / 2$ to $3 / 2$ of the SM value.

\section{The decoupling limit of $2 \mathrm{HDMs}$}

A study of the decoupling limit of $2 \mathrm{HDMs}$ has recently been presented in [15] (see also [16]), where the general expressions for the spectrum and couplings of a generic, non-CP violating, 2HDM are derived. In particular, it is shown (see Eqs. (103)-(106) in [15]) that the approach to decoupling of the $h V V, h t \bar{t}^{4}$ and $h h h$ vertices of the lightest 2HDM Higgs state $h$ (of mass $m_{h}$ ) can be parameterized as follows:

$$
\begin{aligned}
& \frac{g_{h V V}^{2}}{g_{H V V}^{(0)} 2} \sim 1-\frac{\hat{\lambda}^{2} v^{4}}{m_{A}^{4}}, \\
& \frac{g_{h t t}^{2}}{g_{H t t}^{(0)} 2} \sim 1+\frac{2 \hat{\lambda} v^{2} \cot \beta}{m_{A}^{2}}, \\
& \frac{\lambda_{h h h}^{2}}{{\lambda_{H H H}^{(0)}}^{2}} \sim 1-\frac{6 \hat{\lambda}^{2} v^{2}}{\lambda m_{A}^{2}},
\end{aligned}
$$

where the suffices $H$ and (0) label the SM quantities, $m_{A}$ is the mass of the CP-odd Higgs boson, $\tan \beta$ is the ratio of expectation values of the up-type and down-type Higgs doublets,

\footnotetext{
${ }^{4}$ The expression for the case of $b$-quarks is obtained from that for $t$-quarks upon the replacement $\cot \beta \leftrightarrow$ $-\tan \beta$.
} 
$\lambda$ and $\hat{\lambda}$ are dimensionless parameters of the 2HDM. Notice that the deviations from the decoupling limit are proportional to $\epsilon=\hat{\lambda} v^{2} / m_{A}^{2}$ and $\epsilon^{2}$ in the case of the couplings to fermions and gauge bosons whereas the self-coupling is proportional to $\epsilon \hat{\lambda} / \lambda$. The possibility that the ratio $\hat{\lambda} / \lambda$ be large allows for the triple-Higgs self-coupling to remain large even when the other couplings are converging to their SM values. We analyzed this possibility by implementing the exact couplings of a generic 2HDM as discussed in [15] and scanning the parameter space in the range $1<\tan \beta<50^{5},-4 \pi<\lambda_{i}<4 \pi$ for all couplings $\lambda_{i}$ defined in [15], $i=1, \ldots 7$. While in the case $\lambda_{6,7}=0$ full analytical constraints can be derived guaranteeing the Higgs potential be bounded [15], in the general scenario, $\lambda_{6,7} \neq 0$, the stability of the potential has to be checked numerically for every point considered in the parameter space. Our general scan was subject to the constraints of tree-level unitarity [15] and to the requirement that the couplings $g_{h V V}^{2}, g_{h t t}^{2}$ and $g_{h b b}^{2}$ differ from the SM values by no more than $30 \%, 30 \%$ and $70 \%$, respectively. These values reflect the measurement accuracies expected after $300 \mathrm{fb}^{-1}$ of accumulated LHC luminosity $[1]^{6}$. The distribution of $r=\lambda_{H H H} / \lambda_{H H H}^{(0)} \equiv \lambda_{h h h} / \lambda_{H H H}^{(0)}$ for the three Higgs mass values of 120, 130 and $140 \mathrm{GeV}$ in the general case $\left(\lambda_{6,7} \neq 0\right)$ is shown in Fig. 2, where the scan assumed equiprobable input values for all $2 \mathrm{HDM}$ inputs. Some regions of the $2 \mathrm{HDM}$ parameter space can result in small values for $m_{H}$ and $m_{A}$. Hence, we have imposed a further constraint of no visibility at $3 \sigma$ level of the CP-even $H$-state at the LHC through the dominant $g g$ fusion production channel, exploiting the projected significance of ATLAS and CMS for SM Higgs production. The $g g \rightarrow H$ production cross section has been estimated isolating the $b$ - and $t$ - loop contributions in the HIGLU [18] SM predictions and rescaling them with the proper $2 \mathrm{HDM}$ Higgs-quark couplings. As a result of this requirement, all the allowed points in Fig. 2 correspond to $m_{H} \gtrsim 300 \mathrm{GeV}$ and $m_{A} \gtrsim 250 \mathrm{GeV}$. (In the decoupling limit the dynamical behavior of the $A$ state is very similar to that of $H$, so that one can safely assume that whenever the latter is undetectable the former is too.) From now on we shall refer to the region of parameters which survive the above constraints as decoupling limit of the 2HDM. The scan of all $\lambda_{i}$ leads to models with values of $r$ in the ranges ${ }^{7}$

$$
-8 \lesssim r \lesssim 36, \quad M_{H}=120 \mathrm{GeV},
$$

\footnotetext{
${ }^{5}$ Note that in the scans we have traded the Higgs mixing angle $\alpha$ for the lightest Higgs boson mass, $M_{H}$, as independent 2HDM input parameter.

${ }^{6}$ Also a more optimistic scenario of $20 \%, 20 \%$ and $30 \%$ of measurement accuracies [17] has been investigated, yielding conclusions similar to the ones outlined below, though over a restricted parameter range (see also next footnote).

${ }^{7}$ In the case of the more optimistic uncertainty scenario we get $-3.5 \lesssim r \lesssim 18,-3 \lesssim r \lesssim 17$ and $-2 \lesssim r \lesssim 16$ for $M_{H}=120,130$ and $140 \mathrm{GeV}$, respectively. Notice that we did not include so far constraints on $g_{h \tau \tau}^{2}$, because a full detector simulation for this case is still lacking, unlike the other cases. However, for completeness, we quote here some numbers also in presence of $g_{h \tau \tau}^{2}$ limits, though we will not adopt them in the remainder of the analysis. With a projected $10 \%$ accuracy on the latter, hence assuming 30,30 and $10 \%$ as constraints in the scan (with the error on $g_{h \tau \tau}^{2}$ replacing the one on $g_{h b b}^{2}$ ), the above numbers change to $-1.5 \lesssim r \lesssim 18.6,-1.6 \lesssim r \lesssim 34.2$ and $-1.2 \lesssim r \lesssim 12.5$ for $M_{H}=120,130$ and $140 \mathrm{GeV}$, respectively. Assuming 20, 20 and $10 \%$ accuracies, the most optimistic case of all, we have $-1.5 \lesssim r \lesssim 4.1,-1.6 \lesssim r \lesssim 12.4$ and $-1.2 \lesssim r \lesssim 12.1$ for $M_{H}=120,130$ and $140 \mathrm{GeV}$, respectively. Hence, as it will be clear from the following kinematical analysis, whichever the final outcome of the LHC analyses of single-Higgs production channels, all described scenarios for $r$ will still be testable via double-Higgs production.
} 


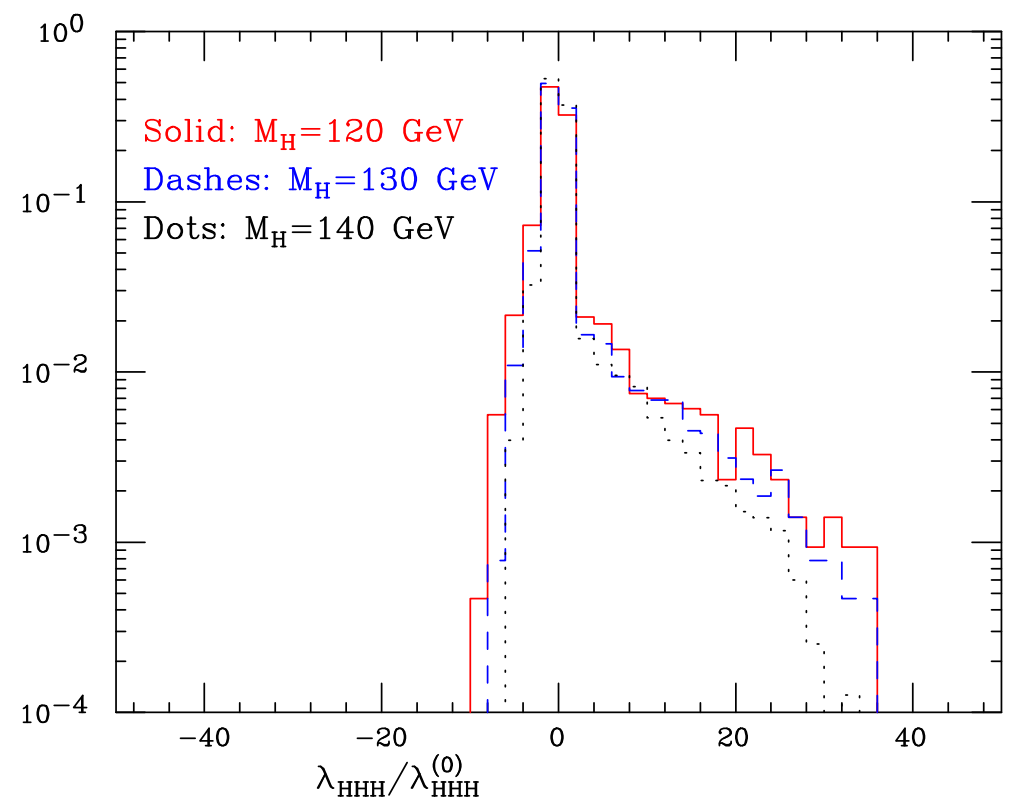

Figure 2: Distribution of the values of $r=\lambda_{H H H} / \lambda_{H H H}^{(0)} \equiv \lambda_{h h h} / \lambda_{H H H}^{(0)}$ in the scans of the $2 \mathrm{HDM}$ parameters space defined in the text, for three values of the lightest Higgs boson mass. Normalization is to unity.

$$
\begin{aligned}
-7 \lesssim r \lesssim 35, & M_{H}=130 \mathrm{GeV}, \\
-6 \lesssim r \lesssim 34, & M_{H}=140 \mathrm{GeV} .
\end{aligned}
$$

\section{Triple-Higgs self-couplings at the LHC}

This Section describes the general setup of our parton-level studies of signal and background rates. The numerical results are obtained by setting the renormalization and factorization scales to $2 M_{H}$ for the vector-vector fusion and Higgs-strahlung signals and to $\sqrt{\hat{s}}$ for heavyquark associated production, with the latter choice accounting for the effect of the large top mass. For the various QCD backgrounds, the factorization/renormalization scale was the average jet $E_{T}$ for the production of light jets and $\sqrt{\hat{s}}$ for final states involving top quarks. Both Higgs processes and QCD backgrounds were estimated by using the parton distribution function (PDF) set MRST99(COR01) [19]. Both signal and background estimates were based on exact tree-level matrix element calculations using the ALPGEN program [20]. Furthermore, all signal rates have been cross-checked by using independent programs, based on either the HELAS subroutines [21] (for $q q^{\left({ }^{(}\right)} \rightarrow q q^{\left({ }^{\prime}\right)} H H$ and $q \bar{q}^{\left({ }^{\prime}\right)} \rightarrow V H H$ ) or the HELAC/PHEGAS package [22] (for $g g \rightarrow t \bar{t} H H$ ). As for numerical input values of SM parameters, we adopt the ALPGEN defaults. We assume that $b$-quark jets are distinguishable from light-quark and gluon ones. Unless otherwise stated, their tagging efficiency (including jet identification) is taken as $\epsilon_{b}=50 \%$ for each $b$ with $E_{T}^{b}>30 \mathrm{GeV}$ and $\left|\eta^{b}\right|<2.5$ $\left(\epsilon_{b}=0\right.$ otherwise) and we also require all light- and $b$-jets to be separated, $\Delta R_{b b, b j, j j}>0.7$. We do not assume $b$-jet charge determination. Finally, in our parton-level analysis, we identify jets with partons. 

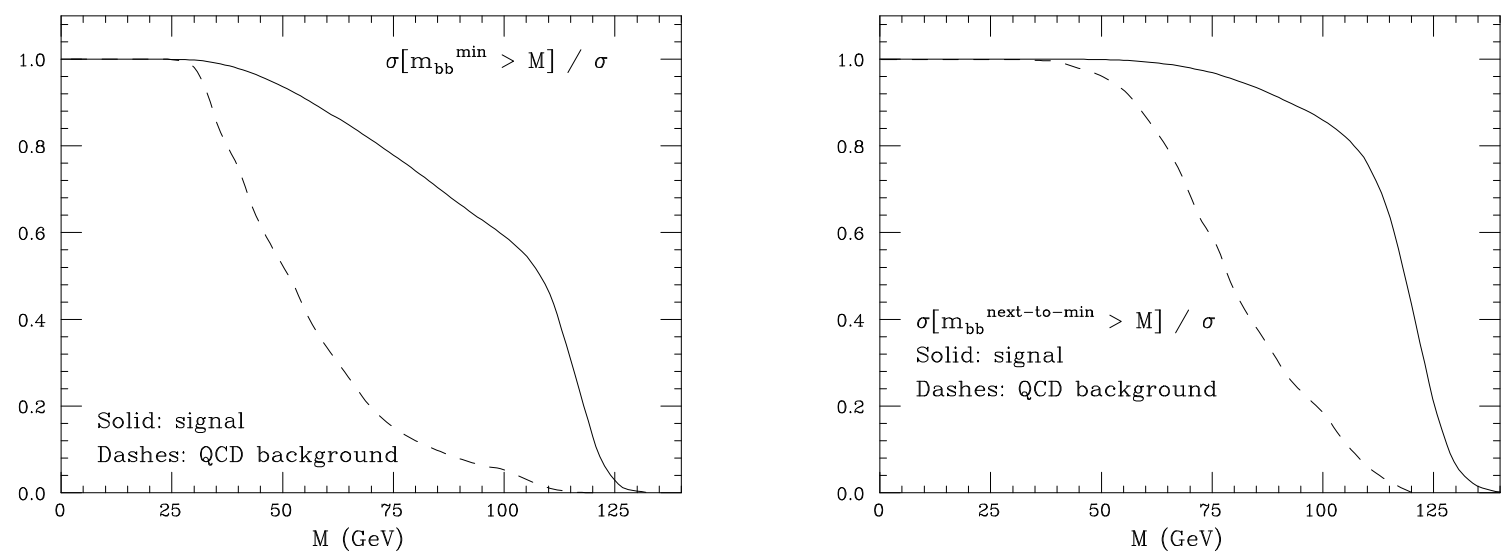

Figure 3: Shapes of the integrated distributions of the minimum (left) and next-to-minimum (right) $b b$ invariant masses in vector-vector fusion and relative QCD background, after the application of the cuts discussed in the text up to Eq. (4.1). Both signal and noise are shown for the case $M_{H}=120 \mathrm{GeV}$.

For the sake of definiteness, the signal results quoted in this Section will refer to the case of SM values for $\lambda_{H H H}$. The analysis of the results in the context of our 2HDM example will be given in the next Section.

\subsection{Vector-vector fusion}

As seen in Fig. 1, within the SM, vector-vector fusion into Higgs boson pairs is the subprocess displaying the second largest cross section for any Higgs mass, behind $g g \rightarrow H H$, and is the most sensitive to deviations of the $\lambda_{H H H}$ coupling from its SM value. In this channel, before any acceptance and selection cuts (and $b$-tagging efficiency) one has production rates of about $1.4(0.7)[0.25] \mathrm{fb}$ for $M_{H}=120(130)[140] \mathrm{GeV}$. If one assumes efficient forward/backward jet tagging and high-purity sampling of $b$-quarks, the dominant background is the QCD production of $b \bar{b} b \bar{b} j j$, where the two extra jets are required to have $E_{T}^{j}>20 \mathrm{GeV}$ and to go one in the forward $\left(\eta^{j}>2.5\right)$ and one in the backward $\left(\eta^{j}<-2.5\right)$ direction. To enforce the reconstruction of the two Higgs bosons, we require all four $b$ 's in the event to be tagged and that at least one out of the three possible double pairings of $b$-jets satisfies the following mass constraint:

$$
\left(m_{b_{1}, b_{2}}-M_{H}\right)^{2}+\left(m_{b_{3}, b_{4}}-M_{H}\right)^{2}<2 \sigma_{m}^{2}
$$

where $\sigma_{m}=0.12 M_{H}$. The $b \bar{b} b \bar{b} j j$ cross sections with this event selection are 98(110)[130] fb for $M_{H}=120(130)[140] \mathrm{GeV}$ (with no $b$-tagging efficiency). The QCD background is dominated by configurations where the two $b$-quark pairs are produced by the splitting of recoiling virtual gluons. These gluons tend to be off-shell by the smallest possible amount, compatibly with the requirement of $\Delta R$ separation and minimum $E_{T}$ of the $b$-jets. The fake Higgs masses are most often reconstructed by pairing $b$ 's travelling back-to-back in the transverse plane, namely pairs coming from the splitting of different gluons. As a result, we expect that even for events where two pairs exist passing the Higgs mass constraint, there 
will be pairings of the four $b$ 's with rather low invariant mass. A very effective way to suppress the QCD background is therefore to cut on the minimum and and next-to-minimum $b \bar{b}$ invariant masses, out of the six possible di-jet pairings, as can be appreciated from Fig. 3 . The dependence of the signal and background cross sections on these additional constraints on $m_{b b}$ masses, also including a more stringent cut on the $E_{T}$ of the forward/backward jets, are displayed in Tab. 1. For our forthcoming numerical investigation on the sensitivity to anomalous triple-Higgs self-couplings we use the kinematic efficiency obtained with event selection c) of Tab. 1. The dependence of both signal and QCD background on the transverse energy of the forward/backward jets can be seen in Fig. 4, e.g., after the cuts in a) of Tab. 1. Other potential background processes, such as $t \bar{t} b \bar{b}, t \bar{t} Z \rightarrow t \bar{t} b \bar{b}, t \bar{t} H \rightarrow t \bar{t} b \bar{b}$, $j j Z Z \rightarrow j j b \bar{b} b \bar{b}$ and $j j b \bar{b} Z \rightarrow j j b \bar{b} b \bar{b}$, have been found to altogether have cross section lower than $10^{-1} \mathrm{fb}$ for event selection c) of Tab. 1 and have thus been neglected in the remainder of our study. The same is true for the process $p p \rightarrow b \bar{b}+4$ light jets, where two light jets are mistagged as $b$-jets with a fake efficiency of 0.01 .

Since in a realistic data analysis the background needs to be measured from the data, the question arises if the stringent cuts applied in the present analysis could produce peaking behaviours of the backgrounds in the signal region. To this aim we studied, for the most relevant background $p p \rightarrow 4 b+2$ forward jets, the handle offered by the distribution

$$
\frac{d \sigma}{d M}, \text { with } M=\sqrt{\left(m_{i 1, i 2}-M_{H}\right)^{2}+\left(m_{i 3, i 4}-M_{H}\right)^{2}}
$$

and $\{i 1, i 2\},\{i 3, i 4\}$ the two pairings minimizing $M$. For the case $M_{H}=120 \mathrm{GeV}$, the cut of Eq. (4.1) implies a cut $M \lesssim 20 \mathrm{GeV}$. The distribution $d \sigma / d M$ (see Fig. 5) does indeed exhibit a strongly peaked structure. However the peak of the distribution is outside the signal region at $120 \mathrm{GeV}$. We therefore expect that the background can be measured by looking at mass intervals away from the latter. As a cross-check of the accuracy of the extrapolation and as an assessment of the induced systematics, one can do the same exercise with a different nominal Higgs mass $\tilde{M}$, away from the signal region, for instance $200 \mathrm{GeV}$ (see also Fig. 5). This allows also the investigation of the region $M \leq \tilde{M} \sqrt{2} \sigma$.

Finally, concerning the trigger, in addition to the forward/backward jets, we have verified that more than $70 \%$ of the signal events are characterized by at least one $b$-jet with $E_{T}>100 \mathrm{GeV}$, which could give an additional handle in selecting Higgs-pair production via vector-vector fusion.

\subsection{Higgs-strahlung}

In the Higgs-strahlung channel, the cross section without any cuts and tagging efficiencies, before the decays of the $W^{ \pm}$and $Z$ bosons, but including the $H H \rightarrow b \bar{b} b \bar{b}$ decay rate, is $0.41(0.19)[0.065] \mathrm{fb}$ for $M_{H}=120(130)[140] \mathrm{GeV}$. Let us first consider the case of leptonic decays of the gauge bosons, with $\ell=e, \mu$. The cuts on $b$-jets used here are the same as for the vector-vector fusion case, with the exclusion of the constraints discussed in Tab. 1. $Z H H$ events are selected requiring two charged leptons with $E_{T}^{\ell}>20 \mathrm{GeV}$, $\left|\eta^{\ell}\right|<2.5$ and invariant mass within $5 \mathrm{GeV}$ of the $Z$ mass. $W^{ \pm} H H$ events are extracted by isolating one charged lepton with $E_{T}^{\ell}>20 \mathrm{GeV}$ and $\left|\eta^{\ell}\right|<2.5$ plus $E_{T}^{\text {miss }}>20 \mathrm{GeV}$. The 

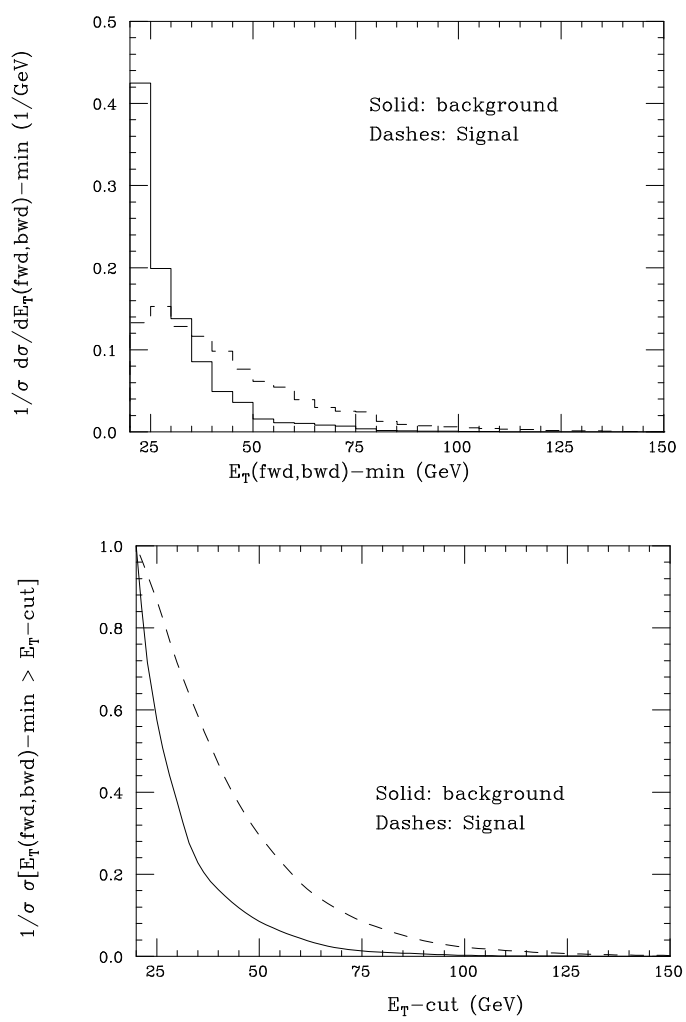

Figure 4: Shapes of the differential (up) and integrated (down) distributions of the minimum forward/backward jet transverse momentum in vector-vector fusion and relative QCD background, after the application of the cuts discussed in the text up to Eq. (4.1) Both signal and noise are shown for the case $M_{H}=120 \mathrm{GeV}$.
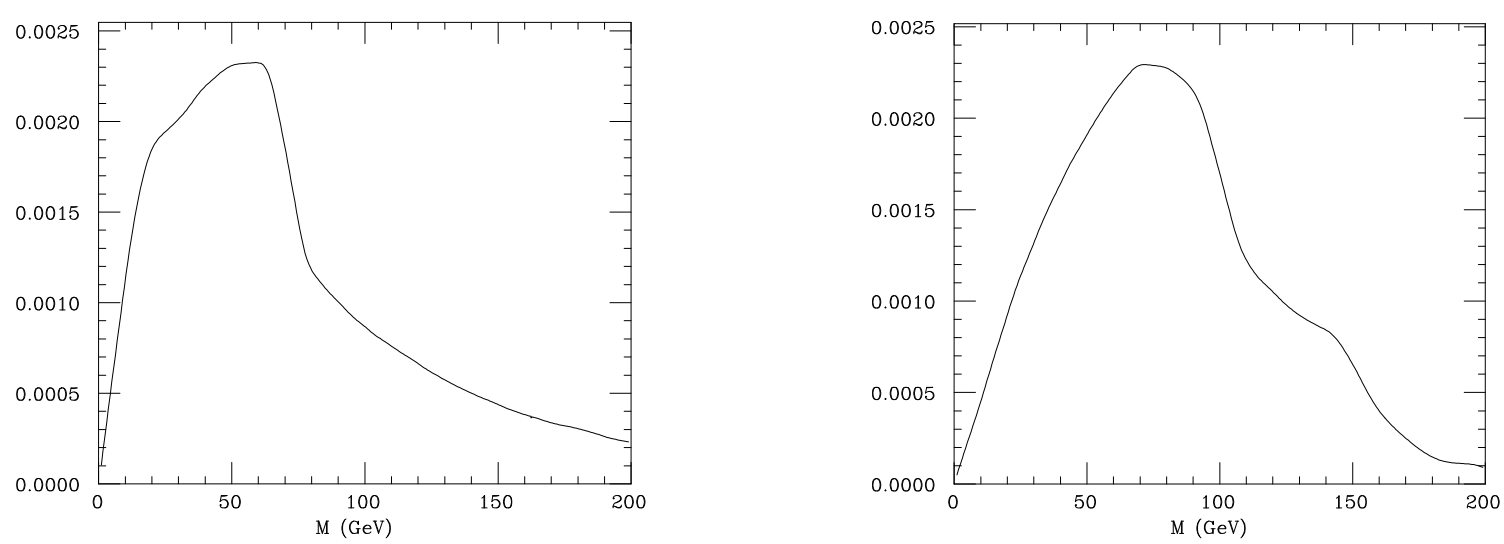

Figure 5: Shape of the distribution of $M$ in Eq. (4.2) for the case $M_{H}=120 \mathrm{GeV}$ (left) and $M_{H}=200 \mathrm{GeV}$ (right).

resulting (cumulative) signal amounts to $0.0083(0.0048)[0.0019] \mathrm{fb}$, for $M_{H}=120(130)[140]$ $\mathrm{GeV}$, prior to $b$-tagging efficiency. The corresponding irreducible background arises from $V b \bar{b} b \bar{b}$ events, with a rate of about $0.5 \mathrm{fb}$ for the above cuts, with very small Higgs mass dependence. We also considered hadronic decays of both $W^{ \pm}$and $Z$, yielding a $4 b$ plus 
2(light-)jets signature. Acceptance, separation and mass cuts on the $4 b$ system are as usual, whereas we require the two light jets to have $E_{T}^{j}>20 \mathrm{GeV},\left|\eta^{j}\right|<2.5$ and to satisfy $70 \mathrm{GeV}<m_{j j}<100 \mathrm{GeV}$. The signal rates increase by a factor of about 4 with respect to the leptonic case, but the irreducible background increases by a factor of about 10. Moreover, here, one should also include the large contribution coming from QCD production of $j j b \bar{b} b \bar{b}$, rendering the channel completely hopeless.

\begin{tabular}{|l|l|l|l|}
\hline$M_{H}(\mathrm{GeV})$ & 120 & 130 & 140 \\
\hline Signal a) (fb) & 0.085 & 0.049 & 0.021 \\
Background a) (fb) & 40 & 35 & 32 \\
\hline Signal b) (fb) & 0.073 & 0.043 & 0.018 \\
Background b) (fb) & 21 & 18 & 17 \\
\hline Signal c) (fb) & 0.036 & 0.021 & 0.008 \\
Background c) (fb) & 4 & 3.5 & 3 \\
\hline
\end{tabular}

Table 1: The effects of additional cuts with respect to those described in the text up to Eq. (4.1) on the signal and background cross sections. Selection a) means $m_{b b}^{\min }>50 \mathrm{GeV}$ and $m_{b b}^{\text {next-to }- \text { min }}>$ $100 \mathrm{GeV}, \mathrm{b})$ corresponds to $m_{b b}^{\min }>70 \mathrm{GeV}$ and $m_{b b}^{\text {next-to-min }}>110 \mathrm{GeV}$ and $\mathrm{c}$ ) to the latter with the more stringent cut $E_{T}^{j}>40 \mathrm{GeV}$ (instead of $E_{T}^{j}>20 \mathrm{GeV}$ ) on the forward/backward jets. No $b$-tagging efficiency is included.

\subsection{Heavy-quark associate production}

As intimated already, in the SM only the case $Q=t$ in Eq. (2.2) has some phenomenological importance. Taking our usual $H H \rightarrow b \bar{b} b \bar{b}$ signature leads to final states which include six $b$-quarks, two of them coming from the decays of $t$ and $\bar{t}$. The largest backgrounds arise from the QCD production of $t \bar{t} b \bar{b} b \bar{b}$ and, if the request of tagging were limited to four $b$-jets only, $t \bar{t} b \bar{b} j j$, where $j$ represents a jet of gluons or light quarks. We estimated the $t \bar{t} b \bar{b} j j$ background and obtained a rate of $1.5 \mathrm{pb}$, with usual jet acceptance cuts $\left(E_{T}^{b, j}>20\right.$ $\left.\mathrm{GeV},\left|\eta^{b, j}\right|<2.5\right)$ and somewhat relaxed jet isolation requirements $\left(\Delta R_{b b, b j, j j}>0.4\right)$.

This implies that tagging all six jets is mandatory. With the above cuts, the $t \bar{t} b \bar{b} b \bar{b}$ background is in fact only $3.6 \mathrm{fb}$. Notice that, for this channel, maintaining a high $b$-tagging efficiency is crucial, since it appears with the sixth power. If the SLHC luminosity led to a decrease from $50 \%$ to $40 \%$, one would loose a factor of 4 in rate, and most of the advantage of the high luminosity gain would be lost. Notice also that in order to keep the $t \bar{t} b \bar{b} j j$ background below the level of $t \bar{t} b \bar{b} b \bar{b}$ requires the fake tagging rate to be kept below $5 \%$ for each jet. To complete the reduction of the continuum background, we require that at least one out of all pairings of $b$-quarks satisfies the constraint given in Eq. (4.1). We consider a semi-leptonic signature with one $W^{ \pm}$decaying to leptons $(e, \mu$ and their neutrinos) and the other one hadronically. So the final states are of the kind $b \bar{b} b \bar{b} b \bar{b} j j \ell \nu_{\ell}$. Events are selected imposing $E_{T}^{j}>20 \mathrm{GeV},\left|\eta^{j}\right|<2.5, \Delta R_{j b, j j}>0.4$. We do not impose $E_{T}, \eta$ or $\Delta R_{\ell j, \ell b}$ criteria on leptons from $W^{ \pm}$decay. The pre-selection criteria for $b$-quarks are $E_{T}^{b}>30 \mathrm{GeV},\left|\eta^{j}\right|<2.5, \Delta R_{b b}>0.7$. With the above cuts, we obtain the signal cross sections $0.021,0.010$ and $0.0035 \mathrm{fb}$ (without including the branching ratio for the $t \bar{t}$ semileptonic signature, $8 / 27$, but including the $\left.\operatorname{BR}(H \rightarrow b \bar{b})^{2}\right)$, corresponding to $M_{H}=120$, 130 and $140 \mathrm{GeV}$, respectively. The irreducible background $t \bar{t} b \bar{b} b \bar{b}$ gives a cross section of $0.007 \mathrm{fb}$ for any relevant Higgs mass. Both signal and background are expected to be sensitive to the choice of factorization/normalization scale, as they originate from QCD induced processes, primarily via gluon PDFs. Hence, we have checked the dependence of these numbers against variations of the factorization/normalization scale. For example, if 
$Q^{2}=\left(2 m_{t}+2 M_{H}\right)^{2}$, we obtain instead the following signal cross sections: $0.025,0.012$ and $0.004 \mathrm{fb}$ for $M_{H}=120,130$ and $140 \mathrm{GeV}$, respectively, while the corresponding background rate grows up to $0.013 \mathrm{fb}$. Detailed studies will be needed to assess to which extent a control data sample can be identified to fix the overall background normalization. The extraction of the signal from backgrounds could be feasible at a $1 \sigma$ statistical level, but the number of events is too small even to see a signal event, as the cross sections quoted above do not include the $6 b$-tagging efficiency factor, i.e., $\epsilon_{b}^{6}$. Assuming $5 b$-tagging instead, always with $\epsilon_{b}=0.5$, the signal events grow by a factor of 7 , at the price of including further background processes: namely, $t \bar{t} b \bar{b}+$ light jets. With the usual fake tagging efficiency of $1 \%$, we checked that there is no sensible gain in statistical significance.

\section{Discussion of the results}

As was clear from the signal and background rates obtained in the previous Section, even the SLHC luminosity option will not be sufficient to establish a statistically significant signal for $H H$ production within the SM in the $4 b$ channel. In this Section we therefore consider the effects of an anomalous $\lambda_{H H H}$ coupling, in the ranges obtained in Section 3. In order to do so, we re-evaluated the signal rates in the presence of non-SM triple-Higgs self-couplings by simply rescaling the value of $\lambda_{H H H}^{(0)}$ to a generic $\lambda_{H H H}$. The corresponding rates, as a function of $\lambda_{H H H} / \lambda_{H H H}^{(0)}$, are shown in Fig. 6. Over the range allowed by our scan and by unitarity, cross section enhancements by up to two orders of magnitude can be obtained. Exclusion limits (at 95\% CL) and signal evidence (at $3 \sigma$ ) for anomalous tripleHiggs self-couplings by combining all channels are found in Tab. 2. In Tab. 3 the limits have been derived taking into account also a forward/backward jet recontruction efficiency of $80 \%$. The by far most sensitive mode is vector-vector fusion, with heavy-quark associated production being of some relevance only for small Higgs masses and positive $\lambda_{H H H} / \lambda_{H H H}^{(0)}$ values, as can be expected by looking at Fig. 6. From the same plot, it is also obvious why the limits obtained via the Higgs-strahlung mode are always much weaker than those extracted in the other two channels.

Notice that the described rescaling is not a gauge invariant operation, because diagrams involving other genuine $2 \mathrm{HDM}$ fields (namely, $H, A$ and $H^{ \pm}$) in the Lagrangian, as well the rescaling of the Higgs-to-SM-particle couplings to the $2 \mathrm{HDM}$ values, are neglected. Therefore, one might suspect that the cross sections in Fig. 6 are anomalously enhanced by unitarity violation effects. To investigate this possibility we have re-computed the vector-vector fusion cross section, including the full set of $2 \mathrm{HDM}$ diagrams, for several hundreds of points uniformly distributed over the $2 \mathrm{HDM}$ parameter space, fulfilling the decoupling conditions of Section 3 and with $r$ in the ranges accessible at the SLHC, namely $r<-2.3(-3.1)[-5.3]$ or $r>3.9(5.9)$ [8.3], in correspondence of $M_{H}=120(130)[140] \mathrm{GeV}$.

As a results of this exercise, we did find points, for any value of $r$, where the 2HDM cross section agrees almost exactly with the one depicted in Fig. 6. Since the calculation is now gauge invariant, this fact alone demonstrates that the approximation implicit in Fig. 6 (namely neglecting additional graphs and coupling modifications) does not lead to an artificial enhancement of the sensitivity to $\lambda_{H H H}$. Furthermore, we found a large 


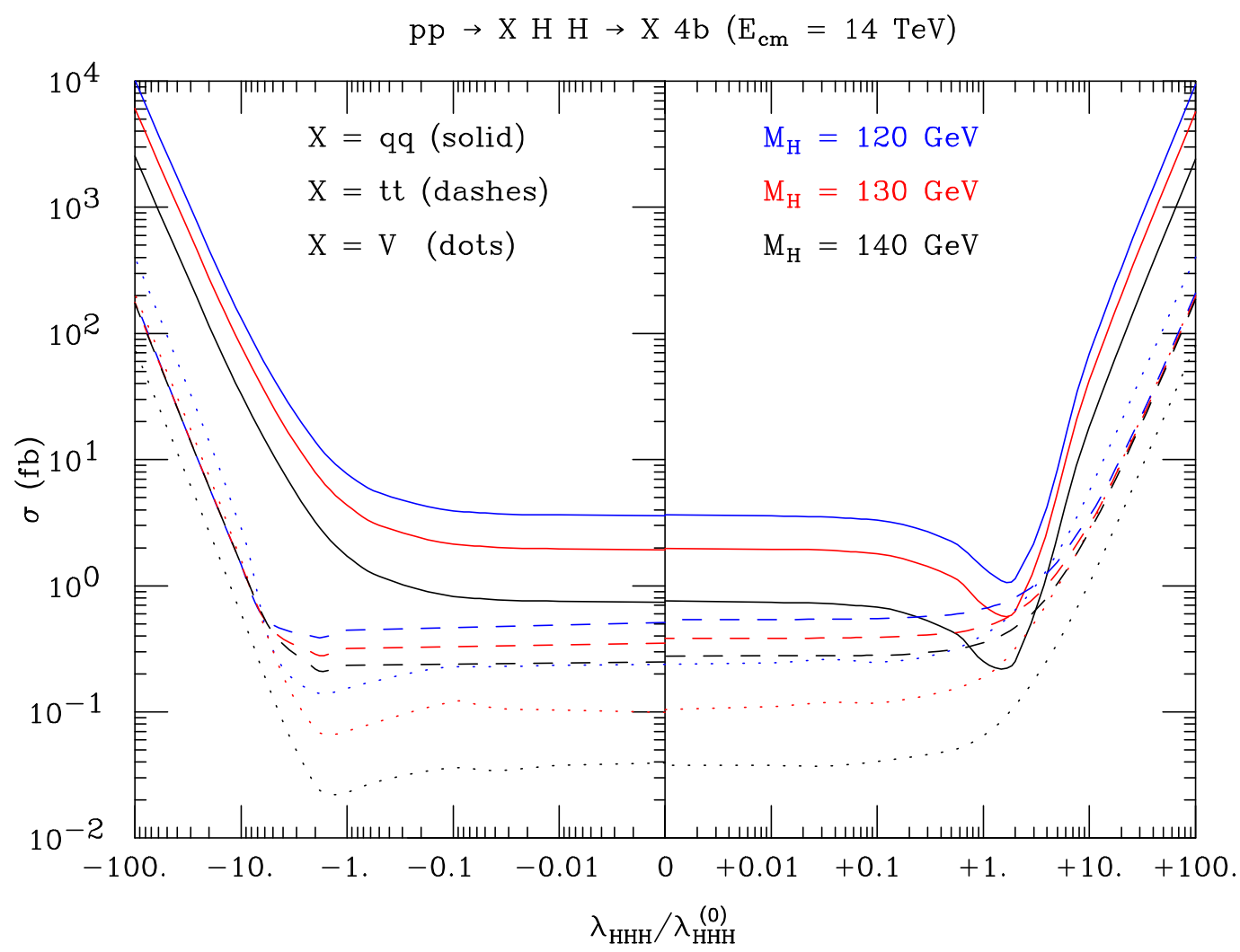

Figure 6: Dependence of the cross sections for the three processes $q q^{\left({ }^{\prime}\right)} \rightarrow q q^{\left({ }^{\prime}\right)} H H, g g, q \bar{q} \rightarrow t \bar{t} H H$ and $q \bar{q}^{\left({ }^{\prime}\right)} \rightarrow V H H$ on $r=\lambda_{H H H} / \lambda_{H H H}^{(0)}$, in correspondence of three values of the Higgs mass, for the model setup discussed in the text.

fraction $(\approx 70 \%)$ of points where the cross section is reproduced by the above described approximation within a factor two. Finally, for a small, but sizeable, portion of points the cross section is substantially underestimated while for even fewer points is grossly overestimated. For completeness, we also tried to understand the origin of such discrepancies. Firstly, the most noticeable ones are due to the $\mathrm{BR}(H \rightarrow b \bar{b})$, as our definition of decoupling region allows for rather sizeable deviations of the latter from the SM value and, since we are looking for two Higgs bosons decaying into $b \bar{b}$, this BR enters quadratically into our predictions. Secondly, also the diagrams proportional to $g_{h V V}(V=W, Z)$ can give an important contribution: although the decoupling limit strongly constraints deviations of $g_{h V V}$ ' from the SM value, the consequences of the latter can be enhanced by large destructive interferences. Once these two effects are accounted for, the overall agreement is fairly good: the contribution of the SM diagrams to the total cross section is correct within a $20 \%$ accuracy, except for a small fraction of points where the production rate is strongly underestimated. We have finally verified that the latter effect is due to neglecting diagrams where a light 2HDM Higgs pair is produced via the decay of the heavy 2HDM Higgs boson, $H \rightarrow h h$. Notice that the effect of this additional contribution reinforces our conclusion that the signal is detectable, actually suggesting that a search is possible even in regions where $\lambda_{H H H}$ exhibits deviations from the SM value smaller than those appearing in Tabs. 2-3. 


\begin{tabular}{|l|rr|rr|rr|}
\hline$M_{H}(\mathrm{GeV})$ & 120 & & 130 & & 140 & \\
\hline LHC, 95\% CL & -4.8 & 7.5 & -6.0 & 9.0 & -9.5 & 12.4 \\
SLHC, 95\% CL & -1.8 & 3.7 & -2.5 & 5.3 & -4.4 & 7.4 \\
\hline LHC, 3 $\sigma$ & -6.6 & 9.3 & -8.1 & 11.0 & -12.4 & 15.4 \\
SLHC, $3 \sigma$ & -2.7 & 5.1 & -3.6 & 6.5 & -5.9 & 8.9 \\
\hline
\end{tabular}

Table 2: Constraints on the ratio $\lambda_{H H H} / \lambda_{H H H}^{(0)}$ using all the channels described in Section 4. (The results are almost completely driven by vector-vector fusion.) In the top box, the two values in each entry correspond to $r_{\min }, r_{\max }$, where $r<r_{\min }$ and $r>r_{\max }$ define the range which can be excluded at 95\% CL (first row) or probed at the $3 \sigma$ level (second row), at both the LHC and SLHC). The number of signal events corresponding to $3 \sigma$ significance are about 120, 110 and 100 for $m_{h} \equiv M_{H}=120,130$ and $140 \mathrm{GeV}$, respectively, at the SLHC for the event selection c) of Tab. 1, with a background of about 1500, 1300 and 1100 events, respectively.

\begin{tabular}{|l|rr|rr|rr|}
\hline$M_{H}(\mathrm{GeV})$ & 120 & & 130 & & 140 & \\
\hline LHC, 95\% CL & -5.6 & 8.0 & -6.9 & 9.8 & -10.8 & 13.7 \\
SLHC, 95\% CL & -2.2 & 3.8 & -3.0 & 5.7 & -5.1 & 8.0 \\
\hline LHC, $3 \sigma$ & -7.6 & 10.0 & -9.2 & 12.0 & -14.1 & 16.9 \\
SLHC, $3 \sigma$ & -3.2 & 5.3 & -4.2 & 7.0 & -6.8 & 9.7 \\
\hline
\end{tabular}

Table 3: The same as in Tab. 2, including a 0.8 reconstruction efficiency for each forward/backward jet.

In closing, we note that our results are not confined to the $2 \mathrm{HDM}$ in the decoupling limit but are truly model independent, thereby being applicable to other, more exotic Higgs sectors displaying a similar decoupling behavior between the lightest CP-even Higgs state and the heavier ones. The study of these models is however beyond the scope of this work.

\section{Summary and outlook}

In the SM, even at the SLHC, the measurement of $\lambda_{H H H}^{(0)}$ for $M_{H} \leq 140 \mathrm{GeV}$ by using subleading Higgs-pair production channels, with the two Higgs particles both decaying into $b \bar{b}$ pairs, appears to be problematic, just like in the case of the leading mode studied in previous literature. However, by studying generic $2 \mathrm{HDM}$ in the decoupling limit, we found large regions of parameter space where the triple-Higgs self-coupling $\lambda_{H H H}$ can differ considerably from the SM value, while keeping all other Higgs interactions with fermions and gauge bosons experimentally consistent with the SM limits. For the allowed regions, where the full $2 \mathrm{HDM}$ result can be emulated in a model-independent way (within an uncertainty of a factor of at worst two) by simply rescaling the SM triple-Higgs selfcoupling to the $2 \mathrm{HDM}$ value, we have analysed the detectability (or otherwise) of various processes of the type $p p \rightarrow X H H \rightarrow X 4 b$ in the mass range $M_{H} \leq 140 \mathrm{GeV}$ at the (S)LHC. In particular, signals at the level of $3 \sigma$ from non-trivial regions of the $2 \mathrm{HDM}$ parameter space can be obtained already at the LHC, with the SLHC extending the scope considerably further. The model-independent approximation used to perform the phenomenological analysis has been thoroughly tested against a complete $2 \mathrm{HDM}$ calculation for the case of 
the vector-vector fusion process, which is the most sensitive to variations of the $\lambda_{H H H}$ coupling. The approximation can be improved (in a model-dependent way, however) by introducing the modified $\mathrm{BR}(H \rightarrow b \bar{b})$ and coupling $g_{H V V}(V=W, Z)$ as predicted in the 2HDM. The only remaining discrepancies can be accounted for, within an accuracy of the order of $30 \%$, by the contribution of the production of a heavy Higgs state and its decay into the light Higgs pair, being the contribution of the remaining non-SM diagrams negligible.

In summary, Higgs-pair production at the (S)LHC could be an important channel to unravel a non-standard Higgs sector. In particular, in the mass window $120 \lesssim M_{H} \lesssim 140 \mathrm{GeV}$, the $4 b X$ signature studied in this document will be the only accessible channel which could show sizeable departures of the trilinear coupling involving the lightest Higgs state from the SM value, in a scenario where the single-Higgs production channels do not show detectable anomalies with respect to the SM predictions.

Finally, our further efforts will concentrate on the case of triple-Higgs self-couplings among neutral Higgs bosons in a general 2HDM away from the decoupling limit as well as in the Minimal Supersymmetric Standard Model (MSSM) (see [26] for 2HDM and MSSM studies of similar vertices involving charged Higgs bosons).

\section{Acknowledgments}

We thank M.L. Mangano for his participation in the early stages of this project and subsequently for many insightful discussions and comments. We also thank F. Boudjema for valuable suggestions, C. Papadopoulos for contributing to the estimate of the heavy-quark associated production cross sections and M. Spira for useful information on the use of the HIGLU programme. SM finally thanks the UK-PPARC for financial support and J. Rathsman for correspondence. ADP thanks the Physics Department of the University of Bari for its kind hospitality. MM and RP acknowledge the financial support of the European Union under contract HPRN-CT-2000-00149. RP also acknowledges the financial support of MIUR under contract 2001023713-006 and of MECD under contract SAB2002-0207. FP wishes to thank the CERN Theory Unit for partial financial support while part of this work was being carried out.

\section{References}

[1] S. Asai et al., SN-ATLAS-2003-024; M. Düehrssen, ATL-PHYS-2003-030; S. Abdullin et al., CMS Note 2003/33.

[2] F. Gianotti, M.L. Mangano and T. Virdee (conveners), hep-ph/0204087.

[3] J.J. van der Bij, Nucl. Phys. B267 (1986) 557.

[4] E.W.N. Glover and J.J. van der Bij, Nucl. Phys. B309 (1988) 282; D.A. Dicus, C. Kao and S.S.D. Willenbrock, Phys. Lett. B203 (1988) 457; G. Jikia, Nucl. Phys. B412 (1994) 57; T. Plehn, M. Spira and P.M. Zerwas, Nucl. Phys. B479 (1996) 46, Erratum, ibidem B531 (1998) 655.

[5] W.-Y. Keung, Mod. Phys. Lett. A10 (1987) 765; O.J.P. Éboli, G.C. Marques, S.F. Novaes and A.A. Natale, Phys. Lett. B197 (1987) 269; D.A. Dicus, K.J. Kallianpur and 
S.S.D. Willenbrock, Phys. Lett. B200 (1988) 187; K.J. Kallianpur, Phys. Lett. B215 (1988) 392; A. Abbasabadi, W.W. Repko, D.A. Dicus and R. Vega, Phys. Rev. D38 (1988) 2770; Phys. Lett. B213 (1988) 386; A. Dobrovolskaya and V. Novikov, Z. Phys. C52 (1991) 427.

[6] V. Barger, T. Han and R.J.N. Phillips, Phys. Rev. D38 (1988) 2766.

[7] A. Djouadi, W. Kilian, M. Mühlleitner and P.M. Zerwas, Eur. Phys. J. C10 (1999) 45.

[8] M. Spira, A. Djouadi, D. Graudenz and P.M. Zerwas, Nucl. Phys. B453 (1995) 17; Z. Kunszt, S. Moretti and W.J. Stirling, Z. Phys. C74 (1997) 479.

[9] R. Lafaye, D.J. Miller, M. Muhlleitner and S. Moretti, hep-ph/0002238.

[10] A. Djouadi et al., in hep-ph/0002258; D. Cavalli et al., in hep-ph/0203056.

[11] E. Richter-Was et al., Int. J. Mod Phys. A13 (1998) 1371; E. Richter-Was and D. Froidevaux, Z. Phys. C76 (1997) 665; J. Dai, J.F. Gunion and R. Vega, Phys. Lett. B371 (1996) 71, ibidem, B378 (1996) 801.

[12] U. Baur, T. Plehn and D. Rainwater, Phys. Rev. D68 (2003) 033001.

[13] U. Baur, T. Plehn and D. Rainwater, Phys. Rev. D69 (2004) 053004.

[14] U. Baur, T. Plehn and D. Rainwater, Phys. Rev. D67 (2003) 033003; Phys. Rev. Lett. 89 (2002) 151801.

[15] J.F. Gunion and H.E. Haber, Phys. Rev. D67 (2003) 075019.

[16] F. Boudjema and A.V. Semenov, Phys. Rev. D66 (2002) 095007; I.F. Ginzburg and M. Krawczyk, hep-ph/0408011; S. Kanemura, Y. Okada, E. Senaha and C.-P. Yuan, hep-ph/0408364; S. Kanemura, Y. Okada, E. Senaha, hep-ph/0410048.

[17] M. Düehrssen et al., hep-ph/0406323 and in hep-ph/0406152.

[18] M. Spira, Nucl. Instrum. Meth. A389 (1997) 357.

[19] See: http://durpdg.dur.ac.uk/hepdata/pdf.html.

[20] M.L. Mangano, M. Moretti, F. Piccinini, R. Pittau and A.D. Polosa, JHEP 0307 (2003) 001.

[21] H. Murayama, I. Watanabe and K. Hagiwara, KEK Report 91-11, January 1992.

[22] A. Kanaki, C.G. Papadopoulos, Comput. Phys. Commun. 132 (2000) 306; C.G. Papadopoulos, Comput. Phys. Commun. 137 (2001) 247.

[23] T. Appelquist and J. Carazzone, Phys. Rev. D11 (1975) 2856.

[24] A. Djouadi, J. Kalinowski and M. Spira, Comput. Phys. Commun. 108 (1998) 56.

[25] S. Moretti and W. J. Stirling, Phys. Lett. B347 (1995) 291, Erratum, ibidem B366 (1996) 451.

[26] S. Moretti and J. Rathsman, Eur. Phys. J. C33 (2004) 41. 